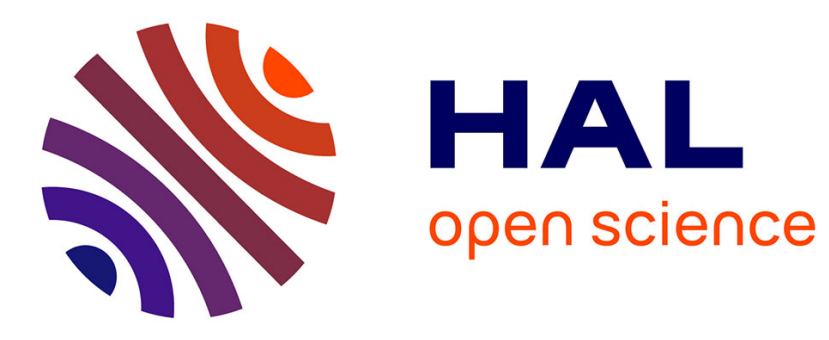

\title{
Generalized Stillinger-Lovett conditions for the one-component plasma
}

\author{
Angel Alastuey
}

\section{To cite this version:}

Angel Alastuey. Generalized Stillinger-Lovett conditions for the one-component plasma. Journal de Physique, 1988, 49 (9), pp.1507-1511. 10.1051/jphys:019880049090150700 . jpa-00210831

\section{HAL Id: jpa-00210831 https://hal.science/jpa-00210831}

Submitted on 1 Jan 1988

HAL is a multi-disciplinary open access archive for the deposit and dissemination of scientific research documents, whether they are published or not. The documents may come from teaching and research institutions in France or abroad, or from public or private research centers.
L'archive ouverte pluridisciplinaire HAL, est destinée au dépôt et à la diffusion de documents scientifiques de niveau recherche, publiés ou non, émanant des établissements d'enseignement et de recherche français ou étrangers, des laboratoires publics ou privés. 


\title{
Generalized Stillinger-Lovett conditions for the one-component plasma
}

\author{
Angel Alastuey \\ Laboratoire de Physique Théorique et Hautes Energies (*), Université Paris-Sud, 91405 Orsay Cedex, France
}

(Reçu le 21 avril 1988, accepté le 20 mai 1988)

\begin{abstract}
Résumé. - Nous établissons un nouvel ensemble de règles de somme concernant les corrélations d'équilibre du plasma classique à une composante. Ces règles peuvent être vues comme des généralisations de la règle usuelle de Stillinger-Lovett relative aux corrélations à deux points. Elles caractérisent le comportement aux grandes longueurs d'onde de la transformée de Fourier (par rapport à un seul point) de la fonction de corrélation de charge à $(n+1)$ points ( $n$ quelconque). Comme la règle de Stillinger-Lovett, les nouvelles règles traduisent des conditions d'écran parfait en phase plasma.
\end{abstract}

\begin{abstract}
We derive a new set of sum rules for the equilibrium correlations of the classical one-component plasma. These sum rules can be viewed as generalizations of the usual Stillinger-Lovett rule refering to the twopoint correlations. They characterize the long-wavelength behaviour of the Fourier transform (with respect to one point) of the $(n+1)$-point charge correlation function for any $n$. Like the Stillinger-Lovett rule, the new rules express perfect screening conditions in a plasma phase.
\end{abstract}

These last years, a large number of sum rules regarding the equilibrium correlations in charged fluids have been derived [1]. Most of these sum rules, like the well-known Stillinger-Lovett rule [2], express the perfect screening of arbitrary external static charges or multipoles. In this paper, we derive a new set of sum rules for the three-point and higher order correlations of a classical one-component plasma (OCP). These sum rules are generalizations of the Stillinger-Lovett condition [2]. They are obtained on the basis of a perfect-screening argument combined to the response theory to all orders. We also indicate how these results can be recovered through a long-wavelength analysis of the generalized Ornstein-Zernike (OZ) equations. The new sum rules are checked in the two-dimensional case, where exact expressions of the $n$-body correlations are available for a special value of the temperature [3]. Finally we briefly mention a possible extension to multicomponent charged fluids with several mobile species immersed in a rigid background.

The OCP is a system of mobile particles with charge $e$ immersed in a rigid neutralizing background with charge density $-e \rho$ if $\rho$ denotes

(*) Laboratoire associé au Centre national de la $\mathrm{Re}$ cherche Scientifique. the mean particle density. The interaction potential between two charges is $e^{2} / r$ in three dimensions, and $-e^{2} \ln (r / L)$ in two dimensions ( $L$ is an irrelevant length scale). Let $\phi(\mathbf{r})$ be the electrostatic potential created by a given distribution $c(\mathbf{r})$ of external charges. In the absence of external charges (i.e. for $c(\mathbf{r})=0)$ and in the fluid plasma phase, the infinite system is homogeneous and the corresponding local charge density $q_{\rho}(\mathbf{r} ; c=0)$ identically vanishes because of overall neutrality. The external charges induce in the infinite system a charge density $q_{\rho}(\mathbf{r} ; c)$ given by

$$
\begin{aligned}
& q_{\rho}(\mathbf{r} ; c)= \\
& \left\langle\exp \left[-\beta \int \mathrm{d} \mathbf{r}^{\prime} Q_{\rho}\left(\mathbf{r}^{\prime}\right) \phi\left(\mathbf{r}^{\prime}\right)\right]\right\rangle_{\rho}
\end{aligned}
$$

where $\beta$ is the inverse temperature, $Q_{\rho}(\mathbf{r})$ is the microscopic charge density operator

$$
Q_{\rho}(\mathbf{r})=e \sum_{j} \delta\left(\mathbf{r}-\mathbf{r}_{j}\right)-e \rho
$$

( $\mathbf{r}_{j}$ is the position of the $j$-th particle), and $\langle\ldots\rangle_{\rho}$ means an equilibrium average over the unperturbed homogeneous infinite system with density $\rho$. The 
perturbative representation of $q_{\rho}(\mathbf{r} ; c)$ in terms of $\phi$ can be readily obtained by rewriting (1) as

$$
\begin{aligned}
q_{\rho}(\mathbf{r} ; c)= & \frac{-1}{\beta} \frac{\delta}{\delta \phi(\mathbf{r})} \ln \left\langle\exp \left[-\beta \int \mathrm{d} \mathbf{r}^{\prime} Q_{\rho}\left(\mathbf{r}^{\prime}\right) \phi\left(\mathbf{r}^{\prime}\right)\right]\right\rangle_{\rho} \\
= & -\frac{1}{\beta} \sum_{n=1}^{\infty} \frac{1}{n !} \int \mathrm{d} \mathbf{r}_{1} \ldots \mathrm{d} \mathbf{r}_{n} \phi\left(\mathbf{r}_{1}\right) \ldots \phi\left(\mathbf{r}_{n}\right) \\
& \times\left.\frac{\delta^{(n+1)}}{\delta \phi(\mathbf{r}) \delta \phi\left(\mathbf{r}_{1}\right) \ldots \delta \phi\left(\mathbf{r}_{n}\right)} \ln \left\langle\exp \left[-\beta \int \mathrm{d} \mathbf{r}^{\prime} Q_{\rho}\left(\mathbf{r}^{\prime}\right) \phi\left(\mathbf{r}^{\prime}\right)\right]\right\rangle_{\rho}\right|_{\phi=0} \\
= & \sum_{n=1}^{\infty} \frac{(-1)^{n}}{n !} \beta^{n} \int \mathrm{d}_{1} \ldots \mathrm{d} \mathbf{r}_{n} \phi\left(\mathbf{r}_{1}\right) \ldots \phi\left(\mathbf{r}_{n}\right) S_{\rho}^{(n+1)}\left(\mathbf{r}, \mathbf{r}_{1}, \ldots, \mathbf{r}_{n}\right)
\end{aligned}
$$

where the last line follows from the definition of the fully truncated $(n+1)$-point charge correlation function $S_{\rho}^{(n+1)}\left(\mathbf{r}, \mathbf{r}_{1}, \ldots, \mathbf{r}_{n}\right)$ of the homogeneous infinite system,

$$
\begin{aligned}
S_{\rho}^{(n+1)}\left(\mathbf{r}, \mathbf{r}_{1}, \ldots, \mathbf{r}_{n}\right) & =\left.\frac{(-1)^{n+1}}{\beta^{n+1}} \frac{\delta^{(n+1)}}{\delta \phi(\mathbf{r}) \delta \phi\left(\mathbf{r}_{1}\right) \ldots \delta \phi\left(\mathbf{r}_{n}\right)} \ln \left\langle\exp \left[-\beta \int \mathrm{d} \mathbf{r}^{\prime} Q_{\rho}\left(\mathbf{r}^{\prime}\right) \phi\left(\mathbf{r}^{\prime}\right)\right]\right\rangle_{\rho}\right|_{\phi=0} \\
& =\left\langle Q_{\rho}(\mathbf{r}) Q_{\rho}\left(\mathbf{r}_{1}\right) \ldots Q_{\rho}\left(\mathbf{r}_{n}\right)\right\rangle_{\rho}^{\mathrm{T}} .
\end{aligned}
$$

Let us consider the particular case where $c(\mathbf{r})$ is the superposition of a plane wave $-e \alpha \exp (i \mathbf{k} \cdot \mathbf{r})$ with a small amplitude $\alpha$ and of some local charge distribution $c_{\ell}(\mathbf{r})$ which creates an electrostatic potential $\psi(\mathbf{r})$. In this case $\phi(\mathbf{r})$ reads

$$
\phi(\mathbf{r})=-\frac{2 \pi^{d / 2}}{\Gamma(d / 2)} \frac{e \alpha}{k^{2}} \exp (i \mathbf{k} \cdot \mathbf{r})+\psi(\mathbf{r})
$$

where $d$ is the dimension and $\Gamma(d / 2)$ is the gamma function. Up to the first order in $\alpha$, the perturbative expansion (2) of $q_{\rho}(\mathbf{r} ; c)$ then reduces to

$$
\begin{aligned}
q_{\rho}(\mathbf{r} ; c)= & \sum_{n=1}^{\infty} \frac{(-1)^{n}}{n !} \beta^{n} \int \mathrm{d} \mathbf{r}_{1} \ldots \mathrm{d} \mathbf{r}_{n} \psi\left(\mathbf{r}_{1}\right) \ldots \psi\left(\mathbf{r}_{n}\right) S_{\rho}^{(n+1)}\left(\mathbf{r}, \mathbf{r}_{1}, \ldots, \mathbf{r}_{n}\right) \\
& +\frac{2 \pi^{d / 2}}{\Gamma(d / 2)} \frac{\beta e \alpha}{k^{2}}\left\{\int \mathrm{d} \mathbf{r}_{1} \exp \left(i \mathbf{k} \cdot \mathbf{r}_{1}\right) S_{\rho}^{(2)}\left(\mathbf{r}, \mathbf{r}_{1}\right)\right. \\
& +\sum_{n=1}^{\infty} \frac{(-1)^{n}}{n !} \beta^{n} \int \mathrm{d} \mathbf{r}_{1} \ldots \mathrm{d} \mathbf{r}_{n+1} \exp \left(i \mathbf{k} \cdot \mathbf{r}_{1}\right) \psi\left(\mathbf{r}_{2}\right) \ldots \psi\left(\mathbf{r}_{n+1}\right) \\
& \left.\times S_{\rho}^{(n+2)}\left(\mathbf{r}, \mathbf{r}_{1}, \ldots, \mathbf{r}_{n+1}\right)\right\}+O\left(\alpha^{2}\right) .
\end{aligned}
$$

In the long-wavelength limit $(k \rightarrow 0)$, the plane wave part of the external charge distribution is perfectly screened by the mobile particles, which then respond to $c_{\ell}(\mathbf{r})$ as if they were immersed in a background carrying a charge density $-e(\rho+\alpha)$. In other words, this partial-screening argument means that the whole system including the internal mobile charges, the background with charge density $-e \rho$, and the external charge distribution $c(\mathbf{r})$, becomes equivalent, in the limit $k \rightarrow 0$, to an OCP with the background charge density $-e(\rho+\alpha)$ in the external electrostatic potential $\psi(\mathbf{r})$ created by $c_{\ell}(\mathbf{r})$. Therefore, one has

$$
\lim _{k \rightarrow 0} q_{\rho}(\mathbf{r} ; c)=q_{\rho+\alpha}\left(\mathbf{r} ; c_{\ell}\right)+e \alpha
$$

where the perturbative representation of $q_{\rho+\alpha}\left(\mathbf{r} ; c_{\ell}\right)$ in terms of $\psi$ is given by the expression (2) with $\psi$ in place of $\phi$ and $S_{\rho+\alpha}^{(n+1)}$ in place of $S_{\rho}^{(n+1)}$. Expanding the latter expression in a Taylor series with respect to $\alpha$, one finds

$$
\begin{aligned}
\lim _{k \rightarrow 0} q_{\rho}(\mathbf{r} ; c) & =\sum_{n=1}^{\infty} \frac{(-1)^{n}}{n !} \beta^{n} \int \mathrm{d} \mathbf{r}_{1} \ldots \mathrm{d} \mathbf{r}_{n} \psi\left(\mathbf{r}_{1}\right) \ldots \psi\left(\mathbf{r}_{n}\right) S_{\rho}^{(n+1)}\left(\mathbf{r}, \mathbf{r}_{1}, \ldots, \mathbf{r}_{n}\right)+ \\
& +e \alpha+\alpha \sum_{n=1}^{\infty} \frac{(-1)^{n}}{n !} \beta^{n} \int \mathrm{d} \mathbf{r}_{1} \ldots \mathrm{d} \mathbf{r}_{n} \psi\left(\mathbf{r}_{1}\right) \ldots \psi\left(\mathbf{r}_{n}\right) \frac{\partial}{\partial \rho} S_{\rho}^{(n+1)}\left(\mathbf{r}, \mathbf{r}_{1}, \ldots, \mathbf{r}_{n}\right)+O\left(\alpha^{2}\right) .
\end{aligned}
$$


On another hand, $\lim q_{\rho}(\mathbf{r} ; c)$ can be obtained by $k \rightarrow 0$

taking the limit $k \rightarrow 0$ in (4). The resulting expression must be identical to (6) for any $\alpha$ and any $\psi$. The identification of the linear terms with respect to $\alpha$ leads to

$$
\int \mathrm{d} \mathbf{r}_{1} \exp \left(i \mathbf{k} \cdot \mathbf{r}_{1}\right) S_{\rho}^{(2)}\left(\mathbf{r}, \mathbf{r}_{1}\right) \sim \frac{\Gamma(d / 2)}{2 \pi^{d / 2}} \frac{k^{2}}{\beta}, k \rightarrow 0
$$

and

$$
\begin{aligned}
\int \mathrm{d} \mathbf{r}_{1} \exp \left(i \mathbf{k} \cdot \mathbf{r}_{1}\right) & S_{\rho}^{(n+1)}\left(\mathbf{r}, \mathbf{r}_{1}, \ldots, \mathbf{r}_{n}\right) \sim \\
& \sim \frac{\Gamma(d / 2)}{2 \pi^{d / 2}} \frac{k^{2}}{\beta e} \frac{\partial}{\partial \rho} S_{\rho}^{(n)}\left(\mathbf{r}, \mathbf{r}_{2}, \ldots, \mathbf{r}_{n}\right) \\
k & \rightarrow 0, n \geqslant 2 .
\end{aligned}
$$

Equation (7) is nothing but the usual StillingerLovett rule, while equations (8) constitute a new set of sum rules $\left({ }^{1}\right)$. Expanding the left hand side of (8) in powers of $\mathbf{k}$, we retrieve that $S_{\rho}^{(n+1)}$ does not carry any monopole, dipole and quadrupole (more generally $S_{\rho}^{(n+1)}$ does not carry any multipole if the clustering is exponential [6]). Furthermore the partial second-moment of $S_{\rho}^{(n+1)}$ is found to be

$$
\begin{aligned}
& \int \mathrm{d} \mathbf{r} r^{2} S_{\rho}^{(n+1)}\left(\mathbf{r} ; \mathbf{r}_{1}, \ldots, \mathbf{r}_{n}\right)= \\
& =\frac{-\mathrm{d} \Gamma(d / 2)}{\pi^{d / 2} \beta e} \frac{\partial}{\partial \rho} S_{\rho}^{(n)}\left(\mathbf{r}_{1}, \ldots, \mathbf{r}_{n}\right), n \geqslant 2 .
\end{aligned}
$$

Replacing $S_{\rho}^{(n+1)}$ and $S_{\rho}^{(n)}$ in (9) by their expressions in terms of the Ursell functions $h_{\rho}^{(m)}$, and using

$$
\rho \int \mathrm{d} \mathbf{r}\left(\mathbf{r}-\mathbf{r}_{1}\right)^{2} h_{\rho}^{(2)}\left(\mathbf{r}, \mathbf{r}_{1}\right)=\frac{-\mathrm{d} \Gamma(d / 2)}{\pi^{d / 2} \beta e^{2} \rho}
$$

(which directly follows from (7)), we find recursively

$$
\begin{aligned}
\rho \int \mathrm{d} \mathbf{r} & \left(\mathbf{r}-\mathbf{r}_{1}\right)^{2} h_{\rho}^{(n+1)}\left(\mathbf{r}, \mathbf{r}_{1}, \ldots, \mathbf{r}_{n}\right)= \\
& -\sum_{j=2}^{n}\left(\mathbf{r}_{j}-\mathbf{r}_{1}\right)^{2} h_{\rho}^{(n)}\left(\mathbf{r}_{1}, \ldots, \mathbf{r}_{n}\right) \\
& -\frac{\mathrm{d} \Gamma(d / 2)}{\pi^{d / 2} \beta e^{2} \rho^{n}} \frac{\partial}{\partial \rho}\left[\rho^{n} h_{\rho}^{(n)}\left(\mathbf{r}_{1}, \ldots, \mathbf{r}_{n}\right)\right]
\end{aligned}
$$

for $n \geqslant 2$. The partial second-moment conditions (11) are generalizations of the familiar second-moment condition (10). It is easy to check that the new rules (11), combined to (10) and the perfect-screening rules

(1) These sum rules for arbitrary $n$, have been derived simultaneously and independently by Vieillefosse and Brajon [4]. The particular case $n=2$ has been already treated by Vieillefosse [5] through a manipulation of the BGY equations.

$$
\begin{aligned}
& \rho \int \mathrm{d} \mathbf{r}_{m} h_{\rho}^{(m)}\left(\mathbf{r}_{1}, \mathbf{r}_{2}, \ldots, \mathbf{r}_{m}\right)= \\
& =-(m-1) h_{\rho}^{(m-1)}\left(\mathbf{r}_{1}, \ldots, \mathbf{r}_{m-1}\right), \quad m \geqslant 3 \\
& \rho \int \mathrm{d} \mathbf{r}_{2} h_{\rho}^{(2)}\left(\mathbf{r}_{1}, \mathbf{r}_{2}\right)=-1
\end{aligned}
$$

constitute an ensemble of compatible equations, as it should be.

It is well known [7] that the Stillinger-Lovett rule (7) can be obtained through a long-wavelength analysis of the two-point $\mathrm{OZ}$ equation. In principle, the generalized rules (8) might be also derived through a similar analysis of the $(n+1)$-point $\mathrm{OZ}$ equations. Here, we present such derivations only in the particular cases $n=2$ and $n=3$ (the corresponding derivations for higher values of $n$ become inextricable because of the complex structure of the $\mathrm{OZ}$ equations). Let $K_{\rho}^{(n)}\left(\mathbf{r}_{1}, \ldots, \mathbf{r}_{n}\right)$ be the $n$-point direct correlation function of the homogeneous infinite OCP with density $\rho$. In Fourier space, the three-point $\mathrm{OZ}$ equation reads [8]

$$
\begin{array}{r}
e^{3} \rho^{2} \tilde{S}_{\rho}^{(3)}\left(\mathbf{k}_{2}, \mathbf{k}_{3}\right)=\tilde{S}_{\rho}^{(2)}\left(\left|\mathbf{k}_{2}+\mathbf{k}_{3}\right|\right) \tilde{S}_{\rho}^{(2)}\left(k_{2}\right) \times \\
\times \tilde{S}_{\rho}^{(2)}\left(k_{3}\right)\left[1+\rho^{2} \tilde{K}_{\rho}^{(3)}\left(\mathbf{k}_{2}, \mathbf{k}_{3}\right)\right]
\end{array}
$$

where $\tilde{f}\left(\mathbf{k}_{2}, \ldots, \mathbf{k}_{n}\right)$ generally denotes the Fourier transform of a function $f\left(\mathbf{r}_{1}, \ldots, \mathbf{r}_{n}\right)=$ $f\left(\mathbf{r}_{12}, \ldots, \mathbf{r}_{1 n}\right)$ which is translationally invariant, i.e.

$$
\begin{aligned}
& \tilde{f}\left(\mathbf{k}_{2}, \ldots, \mathbf{k}_{n}\right)=\int \mathrm{d} \mathbf{r}_{2} \ldots \mathrm{d} \mathbf{r}_{n} \times \\
& \quad \times \exp \left[i\left(\mathbf{k}_{2} \cdot \mathbf{r}_{12}+\cdots+\mathbf{k}_{n} \cdot \mathbf{r}_{1 n}\right)\right] f\left(\mathbf{r}_{12}, \ldots, \mathbf{r}_{1 n}\right) .
\end{aligned}
$$

Taking the limit $k_{2} \rightarrow 0$ in (12), and using (7) one finds

$$
\begin{aligned}
\tilde{S}_{\rho}^{(3)}\left(\mathbf{k}_{2}, \mathbf{k}_{3}\right) & \sim \frac{\Gamma(d / 2)}{2 \pi^{d / 2} \rho^{2} \beta e^{3}} \times \\
& \times\left[\tilde{S}_{\rho}^{(2)}\left(k_{3}\right)\right]^{2}\left[1+\rho^{2} \tilde{K}_{\rho}^{(3)}\left(\mathbf{0}, \mathbf{k}_{3}\right)\right] k_{2}^{2}
\end{aligned}
$$

when $k_{2} \rightarrow 0$. The identity [8]

$$
\begin{aligned}
\tilde{K}_{\rho}^{(n+1)}\left(\mathbf{0}, \mathbf{k}_{3}, \ldots, \mathbf{k}_{n+1}\right)= \\
\quad=\frac{\partial}{\partial \rho} \tilde{K}_{\rho}^{(n)}\left(\mathbf{k}_{3}, \ldots, \mathbf{k}_{n+1}\right), \quad n \geqslant 2
\end{aligned}
$$

combined to the two-point $\mathrm{OZ}$ equation gives

$\tilde{K}_{\rho}^{(3)}\left(\mathbf{0}, \mathbf{k}_{3}\right)=-\frac{1}{\rho^{2}}+\frac{e^{2}}{\left[\tilde{S}_{\rho}^{(2)}\left(k_{3}\right)\right]^{2}} \frac{\partial}{\partial \rho} \tilde{S}_{\rho}^{(2)}\left(k_{3}\right)$

Replacing $K_{\rho}^{(3)}\left(\mathbf{0}, \mathbf{k}_{3}\right)$ by (15) in (13), we obtain

$$
\begin{aligned}
& \tilde{S}_{\rho}^{(3)}\left(\mathbf{k}_{2}, \mathbf{k}_{3}\right) \sim \\
& \sim \frac{\Gamma(d / 2)}{2 \pi^{d / 2} \beta e} k_{2}^{2} \frac{\partial}{\partial \rho} \tilde{S}_{\rho}^{(2)}\left(k_{3}\right), \quad k_{2} \rightarrow 0
\end{aligned}
$$


which is nothing but the particular form of (8) for $n=2$ in Fourier space. "We have checked that the rule (8) for $n=3$ can also be recovered through similar manipulations starting from the four-point $\mathrm{OZ}$ equation [8]

$$
\begin{aligned}
\tilde{S}_{\rho}^{(4)}\left(\mathbf{k}_{2}, \mathbf{k}_{3}, \mathbf{k}_{4}\right) & =\frac{1}{e^{4} \rho^{3}} \tilde{S}_{\rho}^{(2)}\left(k_{2}\right) \times \\
& \times \tilde{S}_{\rho}^{(2)}\left(k_{3}\right) \tilde{S}_{\rho}^{(2)}\left(k_{4}\right) \tilde{S}_{\rho}^{(2)}\left(\left|\mathbf{k}_{2}+\mathbf{k}_{3}+\mathbf{k}_{4}\right|\right) \\
& \times\left[\rho^{3} \tilde{K}_{\rho}^{(4)}\left(\mathbf{k}_{2}, \mathbf{k}_{3}, \mathbf{k}_{4}\right)-2\right] \\
& +\tilde{S}_{\rho}^{(3)}\left(\mathbf{k}_{2}, \mathbf{k}_{3}\right) \frac{\tilde{S}_{\rho}^{(3)}\left(\mathbf{k}_{2}+\mathbf{k}_{3}, \mathbf{k}_{4}\right)}{\tilde{S}_{\rho}^{(2)}\left(\left|\mathbf{k}_{2}+\mathbf{k}_{3}\right|\right)} \\
& +\tilde{S}_{\rho}^{(3)}\left(\mathbf{k}_{2}, \mathbf{k}_{4}\right) \frac{\tilde{S}_{\rho}^{(3)}\left(\mathbf{k}_{2}+\mathbf{k}_{4}, \mathbf{k}_{3}\right)}{\tilde{S}_{\rho}^{(2)}\left(\left|\mathbf{k}_{2}+\mathbf{k}_{4}\right|\right)} \\
& +\tilde{S}_{\rho}^{(3)}\left(\mathbf{k}_{3}, \mathbf{k}_{4}\right) \frac{\tilde{S}_{\rho}^{(3)}\left(\mathbf{k}_{3}+\mathbf{k}_{4}, \mathbf{k}_{2}\right)}{\tilde{S}_{\rho}^{(2)}\left(\left|\mathbf{k}_{3}+\mathbf{k}_{4}\right|\right)}
\end{aligned}
$$

It is amusing to check the second-moment conditions (11) in the particular case of the two-dimensional OCP at $\beta e^{2}=2$, where the general (exact) expression of $h_{\rho}^{(n)}$ reads [3]

$$
\begin{array}{r}
h_{\rho}^{(n)}\left(\mathbf{r}_{1}, \ldots, \mathbf{r}_{n}\right)=(-1)^{n+1} \exp \left(-\pi \rho \sum_{j=1}^{n}\left|z_{j}\right|^{2}\right) \times \\
\times \sum_{\left(i_{1} \ldots i_{n}\right)} \exp \left[\pi \rho\left(z_{i_{1}} z_{i_{2}}^{*}+\cdots+z_{i_{n}} z_{i_{1}}^{*}\right)\right] .
\end{array}
$$

In (17), $z_{j}$ denotes the complex number $z_{j}=r_{j} \exp \left(i \theta_{j}\right)$ where $\left(r_{j}, \theta_{j}\right)$ are the polar coordi- nates of $\mathbf{r}_{j}$ in a given frame, $z_{j}^{*}$ is the complex conjugate of $z_{j}$, and the summation $\sum_{\left(i_{1} \ldots i_{n}\right)} \ldots$ runs over all cycles $\left(i_{1}, \ldots, i_{n}\right)$ built with $(1, \ldots, n)$. Let us compute the second moment

$$
\rho \int \mathrm{d} \mathbf{r}\left(\mathbf{r}-\mathbf{r}_{1}\right)^{2} h_{\rho}^{(n+1)}\left(\mathbf{r}, \mathbf{r}_{1}, \ldots, \mathbf{r}_{n}\right)
$$

with the help of the expression (17) of $h_{\rho}^{(n+1)}$. For each cycle $\left(j_{1}, \ldots, j_{n+1}\right)$ built with $(1, \ldots, n, 0)$ and such that $\mathbf{r}_{j_{k}}=\mathbf{r}_{0}=\mathbf{r}$, one has the Gaussian weight factor

$$
\begin{aligned}
\exp \left(-\pi \rho r^{2}\right) \exp \left\{\pi \rho \left[r_{j_{k-1}}\right.\right. & r \exp \left(i \theta_{j_{k-1}}-i \theta\right)+ \\
& \left.\left.+r r_{j_{k+1}} \exp \left(i \theta-i \theta_{j_{k+1}}\right)\right]\right\} .
\end{aligned}
$$

Replacing the second exponential by its Taylor expansion, and using the orthogonality properties of the functions $\exp (\operatorname{im} \theta)$, we find that the contribution of such a cycle to (18) is

$$
\begin{aligned}
& (-1)^{n} \exp \left(-\pi \rho \sum_{j=1}^{n}\left|z_{j}\right|^{2}\right) \\
& \times \exp \left[\pi \rho\left(z_{j_{1}} z_{j_{2}}^{*}+\cdots+z_{j_{k-1}} z_{j_{k+1}}^{*}+\cdots+z_{j_{n+1}} z_{j_{1}}^{*}\right)\right] \\
& \times\left(\frac{1}{\pi \rho}+z_{j_{k-1}} z_{j_{k+1}}^{*}-z_{1}^{*} z_{j_{k-1}}-z_{1} z_{j_{k+1}}^{*}+r_{1}^{2}\right) .
\end{aligned}
$$

Putting together the contributions of the $n$ cycles $\left(j_{1}, \ldots, j_{n+1}\right)$ such that $\left(j_{1}, \ldots, j_{k-1}, j_{k+1}, \ldots, j_{n+1}\right)$ defines a given cycle $\left(i_{1}, \ldots, i_{n}\right)$ built with $(1, \ldots, n)$, we find that (18) reduces to

$$
\begin{aligned}
(-1)^{n} \sum_{j=2}^{n}\left(\mathbf{r}_{j}-\mathbf{r}_{1}\right)^{2} \sum_{\left(i_{1} \ldots i_{n}\right)} \exp \left(-\pi \rho \sum_{j=1}^{n}\left|z_{j}\right|^{2}\right) \exp \left[\pi \rho\left(z_{i_{1}} z_{i_{2}}^{*}+\cdots+z_{i_{n}} z_{i_{1}}^{*}\right)\right]+ & \\
& +(-1)^{n} \sum_{\left(i_{1} \ldots i_{n}\right)} \exp \left(-\pi \rho \sum_{j=1}^{n}\left|z_{j}\right|^{2}\right) \exp \left[\pi \rho\left(z_{i_{1}} z_{i_{2}}^{*}+\cdots+z_{i_{n}} z_{i_{1}}^{*}\right)\right] \\
& \times\left(\frac{n}{\pi \rho}-\sum_{j=1}^{n}\left|z_{j}\right|^{2}+z_{i_{1}} z_{i_{2}}^{*}+\cdots+z_{i_{n}} z_{i_{1}}^{*}\right)
\end{aligned}
$$

which is indeed the right-hand side of (11) computed using (17), $d=2$ and $\beta e^{2}=2$.

We have derived a new set of sum rules for the OCP, which characterize the long-wavelength behaviour of the Fourier-transform with respect to one point of the $(n+1)$-point charge correlation function. This behaviour is of the Stillinger-Lovett type, with a coefficient which depends on the $n$ other points through the partial derivative with respect to the density of the $n$-point charge correlation function. One might derive similar sum rules for multicomponent charged fluids with several mobile species immersed in a rigid homogeneous background ensuring the overall neutrality. The general derivation given above clearly applies to the latter case, if one takes into account properly the variation of density of each species induced by the external charge distribution $-e \alpha$. Consequently, the corresponding sum rules involve partial derivatives with respect to the different species densities (these sum rules are different from those recently derived by Suttorp and Van Wonderen [9]). All these sum rules are expected to hold for any values of the thermodynamic parameters which define a plasma phase. 


\section{References}

[1] For a review see MARTIN, Ph. A., to be published in Rev. Mod. Phys.

[2] Stillinger, F. H. and Lovett, R., J. Chem. Phys. 48 (1968) $3858 ; 49$ (1968) 1991.

[3] Connu, F. and JancovicI, B., Europhys. Lett. 5 (1988) 125.

[4] Vieillefosse, P. and Brajon, M., to be published.

[5] Vieillefosse, P., J. Stat. Phys. 41 (1985) 1015.
[6] Blum, L., Gruber, Ch., Lebowitz, J. L. and MarTIN, Ph. A., Phys. Rev. Lett. 48 (1982) 1769.

[7] See, e.g. Alastuey, A., Ann. Phys. Fr. 11 (1986) 653.

[8] Alastuey, A., Mol. Phys. 52 (1984) 637.

[9] Suttorp, L. G. and Van Wonderen, A. J., Physica 145A (1987) 533. 